\title{
Highly efficient genome editing via 2A-coupled co-expression of two TALEN monomers
}

\author{
Andrew Mariano ${ }^{1}$, Li Xu ${ }^{2}$ and Renzhi Han²
}

\begin{abstract}
Background: Transcription activator-like effector nucleases (TALENS) are a useful tool for targeted gene editing. TALEN monomers are traditionally expressed from two different plasmids. Each encodes a different TALEN arm that binds to a user-defined sequence and mediates gene editing. Expression of TALEN monomers in two separate plasmids requires co-delivery of each plasmid to the cell. Efficacy of gene editing may be increased if each monomer was transcribed from the same reading frame.

Findings: We developed a TALEN scaffold which expresses both TALEN monomers from a single open reading frame in equal molar amount by linking both monomers with a 2A self-cleaving peptide sequence. This TALEN scaffold, named PTAL10, demonstrates higher levels of genome editing than co-transfected TALENs at similar levels of transfection efficiencies when analyzed for TALEN-induced small insertions and deletions.
\end{abstract}

Conclusions: This protocol for gene editing using 2A-linked TALENs requires transfection of only one plasmid as compared to transfection of two separate plasmids encoding each TALEN monomers.

Keywords: Gene editing, TALEN, 2A self-cleaving sequence

\section{Findings \\ Background}

Transcription activator-like effector nucleases (TALENs) are an exciting gene-editing tool that has emerged in recent years. Their DNA-binding domains are based on transcription activator-like effector (TALE) proteins from Xanthomonas plant pathogens [1-4]. The central repeat domain in the TALE structure mediates DNA binding with each repeat specifying one target base. The base preference of each repeat is determined by two critical, adjacent amino acids referred to as the "repeat variable di-residue" (RVD) which preferentially recognizes one of the four bases in the target site $[5,6]$. This simple "two amino acids for one base" code enables rapid engineering of customized TALE repeat arrays that recognize a user-defined target sequence. Using default settings for TAL Effector Nucleotide Targeter 2.0 [7], one can identify a unique TALE-binding site for every nucleotide of target sequence-this frequency makes it a highly attractive gene editing tool. Indeed, TALEN has

\footnotetext{
* Correspondence: renzhi.han@osumc.edu

${ }^{2}$ Department of Surgery, The Ohio State University Wexner Medical Center, Columbus, $\mathrm{OH} 43210$, USA

Full list of author information is available at the end of the article
}

been used to modify genes in a number of different cell types and organisms with low off-target activity [8].

Several approaches have been taken to improve the specificity and efficiency of TALEN, including modification of FokI cleavage domain $[9,10], \mathrm{N}$-terminal and $\mathrm{C}$-terminal segments flanking central repeat domains [11], and enrichment of edited cells by using fluorescence activated cell sorting (FACS) [12]. Two TALEN monomers are required to induce a double strand break in the genomic DNA of the cells, thus requiring co-delivery of both TALEN monomers into the target cells. To reduce the complication associated with co-transfection by which a portion of cells are only singly transfected, we previously [13] demonstrated that two functional TALEN monomers can be expressed from a single transcript by the use of a self-cleaving $2 \mathrm{~A}$ peptide derived from equine rhinitis A virus (E2A; QCTNYALLKLAGD VESNPGP) or Thosea asigna virus (T2A; EGRGSLLT CGDVEENPGP) [14,15]. Upon translation, the ribosome skips the synthesis of the glycyl-prolyl peptide bond between the ending glycine and proline of the $2 \mathrm{~A}$ sequence, resulting in the release of the antecedent protein and the re-initiation of translation of the following proline amino acid [16]. This strategy ensures that each 
transfected cell receives both TALEN monomers at an equal molar amount, ultimately displaying higher gene editing efficiencies.

In the present study, we compared the gene editing performances of previously constructed TALENs targeting two different loci: MSTN [13] and AAVS1 locus [3]. Additionally, we provided a protocol that can help guide construction of a functional TALEN using pTAL10 scaffold.

\section{Results and discussion}

We quantitatively compared the gene editing performance of our previously characterized TALENs targeting myostatin [13] expressed in one plasmid with the same TALENs expressed in two separate plasmids. HEK293 cells were transfected with either mock, co-transfection (myostatin TALEN left and right monomers in two separate plasmids), or pTAL10-MSTN (two TALEN monomers in one plasmid) (Figure 1). Western blotting showed that the TALEN monomers $(\sim 110 \mathrm{kDa})$ were faithfully expressed from either the two separate plasmids (each TALEN monomer is fused with a $3 \times$ FLAG tag) or the single plasmid linked by a T2A sequence (right TALEN monomer is fused with a $2 \times \mathrm{HA}$ tag, whereas the left monomer is fused with a $3 \times$ FLAG tag) (Figure 2a). Consistent with previous reports that T2A mediates high self-cleavage efficiency [15], only a very small fraction of the $220 \mathrm{kDa}$ full-length precursor protein was visible with a high exposure time (Figure 2a). Gene editing activity was quantified by the mismatch-sensitive T7 endonuclease I (T7E1) assay which detects the TALENinduced insertions and deletions (indels) around the target site (Figure $2 \mathrm{~b}$ and $\mathrm{c}$ ). TALENs expressed from one single plasmid exhibited higher gene editing activity than co-transfection of two TALEN monomers under the same conditions (Figure $2 \mathrm{~b}$ and $\mathrm{c}$ ).

To test whether the improved gene editing activity is MSTN specific, we constructed another previously characterized TALENs targeting human AAVS1 locus [17]. Again, the AAVS1-TALEN monomers were faithfully expressed and properly cleaved using the pTAL10 scaffold (Figure 2d). T7E1 assay showed that AAVS1 TALENs expressed from the one-plasmid pTAL10 induced higher gene editing activity than the two separated plasmid system (Figure 2e and f).

To facilitate the assembly of two TALEN monomers into one plasmid, we constructed a TALEN scaffold designated as pTAL10 (Figure 3), wherein two TALEN monomers (left and right) can be sequentially assembled in a similar way as previously described [4] with some modifications. After assembly of user-defined RVD sequences in their respective pFUS backbone, these RVD sequences and last repeat sequence (pLR-X, where $\mathrm{X}$ represents RVD) can be ligated to the left TALEN scaffold of pTAL10 upon digestion with BsmBI (Figure 3, Step 1). To complete construction, Golden Gate assembly is repeated on the pTAL10 containing assembled left TALEN for the right monomer. Different from the previous reaction, this digestion includes both BsmBI and BsaI for unveiling appropriate sticky-ends (Figure 3, Step 2). Each TALEN monomer scaffold is based on the GoldyTALEN [18] which has increased gene editing efficiency.

In order to detect expression of each monomer uniquely by western blotting analysis, each monomer is fused with a tag at its N-terminal: 3xFLAG for left TALEN monomer, and 2xHA for the right. In addition, the TALEN monomer scaffold uses the obligate heterodimeric FokI variants (ELD-sharkey or KKR-sharkey) [13]. The two monomers and GFP (used as a reporter for transfection) are linked by the aforementioned Thosea asigna and equine rhinitis A virus 2A peptide sequences, respectively. We removed all BsaI and BsmBI sites outside of the TALEN scaffold in the final pTAL10 plasmid using site-directed mutagenesis so that it is fully compatible with the Golden Gate platform [4]. The map and sequence of pTAL10 as well as a detailed step-bystep protocol to assemble the TALEN monomers into this single TALEN scaffold plasmid are provided in the supplement (Additional files 1 and 2).

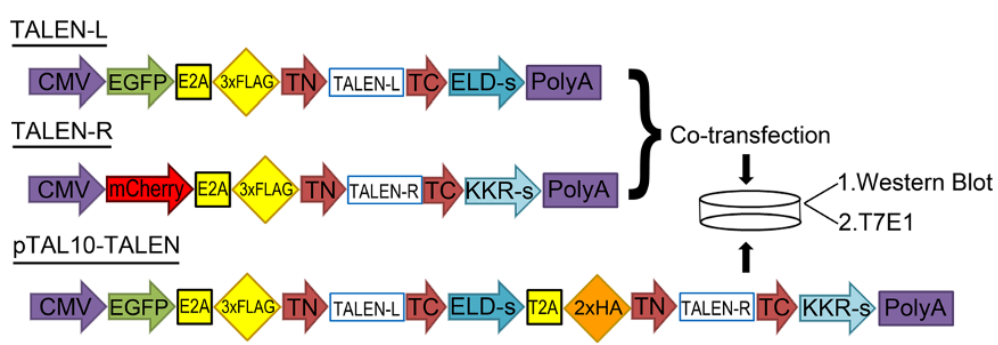

Figure 1 Cartoon of relevant domains for TALEN-L (left), TALEN-R (right), and pTAL10-TALEN. Diagram of notable regions of myostatin or AAVS1 locus targeting TALEN constructs (TALEN-L, TALEN-R, and PTAL 10-TALEN) and experimental design. Monomers TALEN-L and TALEN-R contain EGFP/3xFLAG or mCherry/3xFLAG for transfection and biochemical monitoring respectively. pTAL10-TALEN contains both TALEN monomers, linked by a self-peptide cleaving sequence. Left and right monomers can be fluorescently or biochemically monitored by EGFP/3xFLAG or mCherry/2xHA tags respectively. 


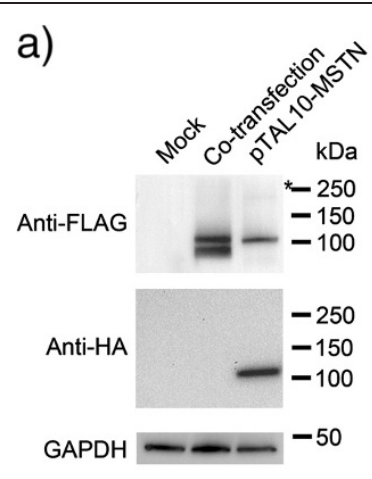

d)

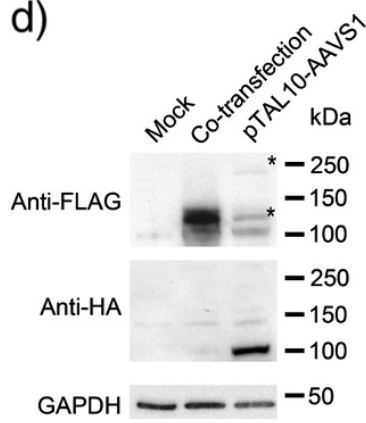

b)

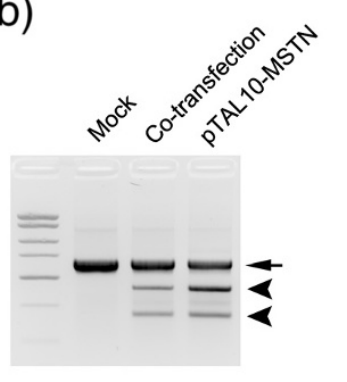

e)

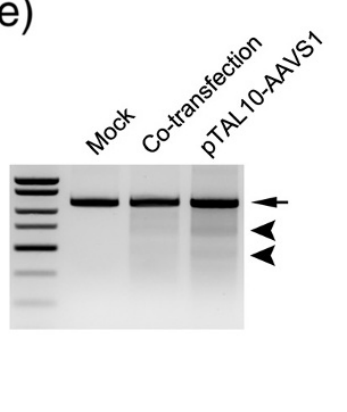

c)

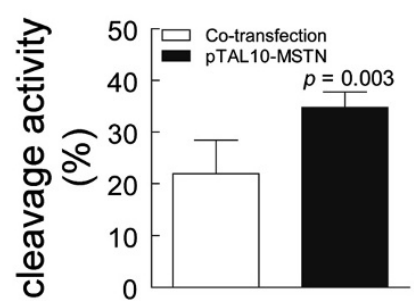

f)

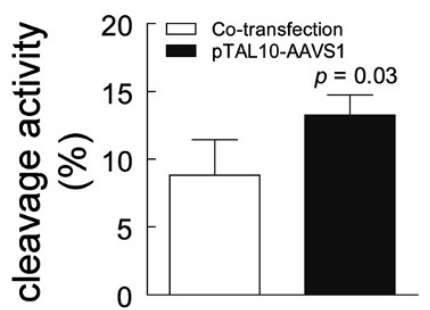

Figure 2 T2A-linked TALENs demonstrate increased gene editing activity than co-transfected TALEN monomers. (a, d) Western blotting shows proper expression of co-transfected TALENs, tagged with 3XFLAG sequence, or pTAL10-TALENs, tagged with 2xHA/3xFLAG sequences, targeting MSTN or AAVS1 respectively. Asterisk $\left(^{*}\right)$ depicts uncleaved TALENs arms or GFP-TALEN. GAPDH was used as a loading control. (b, e) Representative gel of T7E1 assay comparing gene editing activities of co-transfected TALENs and T2A-linked TALENs targeting MSTN or AAVS1 respectively. Upper band, demarcated with an arrow, represents noncleaved DNA; bottom bands, demarcated with an arrow head, represent cleaved DNA fragments. Image is representative of $4-6$ biological replicates. (c, $\mathbf{f}$ Cleavage activity of TALEN (TALEN-L/R co-transfection vs. pTAL10-TALEN) targeting MSTN or AAVS1 respectively were compared using T7E1 endonuclease assay. Results are from 4-6 biological replicates. $p<0.05$ was considered to be significant.

\section{Conclusion}

We have generated an improved TALEN scaffold, designated as pTAL10, by utilizing a self-cleaving peptide sequence. pTAL10 demonstrates higher gene editing when compared to co-transfected TALEN-L and R due to proper co-delivery of TALE monomers. Additionally, this improved scaffold allows transfection efficiency to be fluorescently monitored, while expression of each TALEN arm to be biochemically monitored.

\section{Methods}

Creation of TALE nucleases

Construction of AAVS1-L, AAVS1-R, MSTN-L, and MSTN-R for co-transfection was previously described

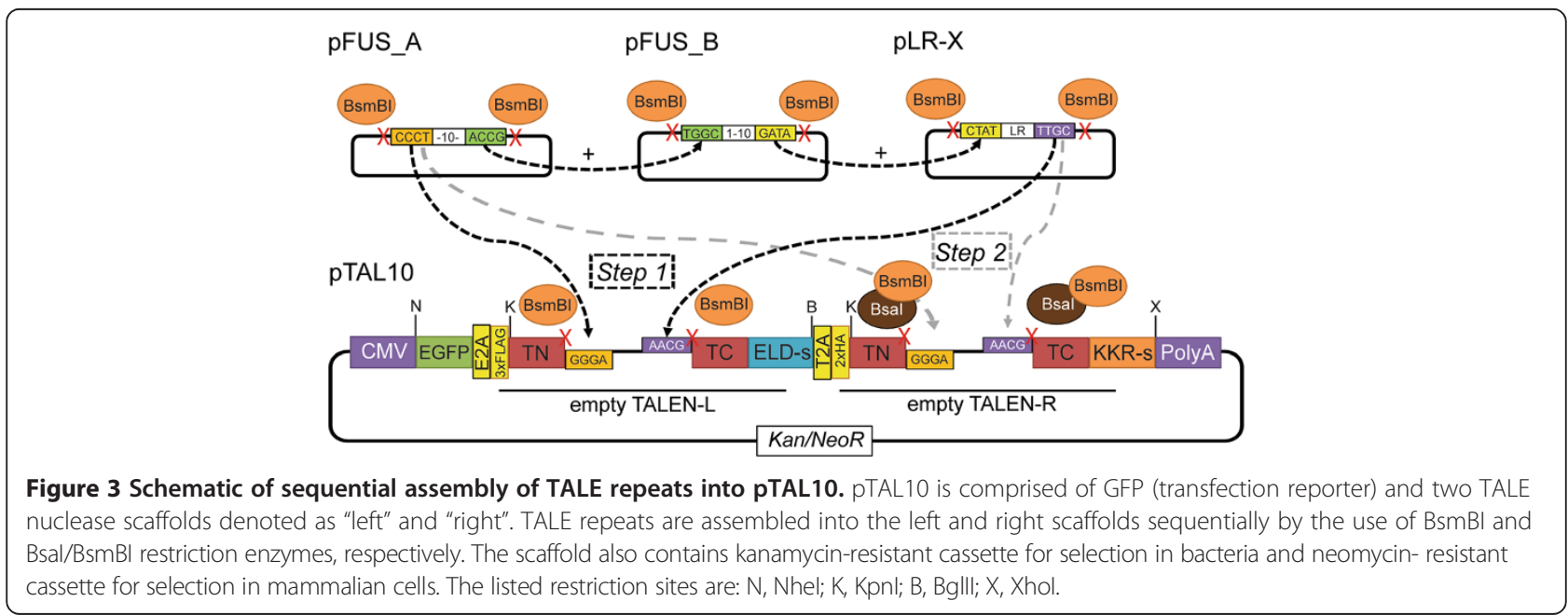


[13]. AAVS1-L/AAVS1-R and MSTN-L/MSTN-R for creation of pTAL10-AAVS1 and pTAL10-MSTN, respectively, was constructed using Golden Gate TALEN Kit 2.0 (Kit \#1000000024) from Addgene. These sequences were then added to pTAL10 (Figure 2) by using BsmBI and $\mathrm{BsmBI} / \mathrm{BsaI}$ digestion respectively. Further details on creation of TALE nucleases using pTAL10 scaffold is discussed below and in the supplemental protocol.

\section{Cell culture and transfection}

HEK293 cells were cultured in DMEM supplemented with 10\% FBS, 1\% L-glutamine (Thermo Scientific Hyclone, Waltham, MA), and 1\% Penicillin-Streptomycin (Thermo Scientific Hyclone, Waltham, MA). Transient transfection by Xfect transfection reagent (Clontech, Mountain View, CA) was done according to manufacturer's recommendation. In short, HEK293 cells for each downstream application (genomic DNA or protein extraction) were seeded in a 6-well plate until $60-70 \%$ confluency. Thereafter, $5 \mu \mathrm{g}$ of total DNA (pTAL10-TALEN single plasmid transfection, or TALEN-L/ TALEN-R co-transfection) in nanoparticle complex solution was added to the culture medium dropwise, followed by replacement of medium 16-20 hours post transfection. After 72 hours, either genomic DNA or protein was extracted from these cell lines. Transfection efficiency was determined by fluorescence microscopy analysis: 1) the average percentage of mCherry- or GFP-positive cells for co-transfection or 2) the percentage of GFP-positive cells for pTAL10-TALENs.

\section{Western blot}

HEK293 transfected with single pTAL10-MSTN or cotransfection with MSTN-L and MSTN-R were lysed 4872 hours post transfection with cold RIPA buffer supplemented with protease inhibitors (1 mM PMSF and $1 \mathrm{mM}$ Benzamidine). Protein concentration was determined by using Bradford Protein Assay Kit (IBI Scientific, Peosta, IA). $20 \mu \mathrm{g}$ of protein were resolved on a 4-20\% MiniPROTEAN TGX Precast Gel (Bio-Rad Laboratories, Hercules, CA) and transferred onto a PVDF membrane (Millipore, Billerica, MA). Immunoblotting was done with the following antibodies: mouse monoclonal anti-FLAG (F3165; Sigma-Aldrich, Saint Louis, MO), polyclonal anti-HA-Tag antibody (Clontech, Mountain View, CA), mouse monoclonal anti-GAPDH (Millipore, Billerica, MA), and HRP conjugated goat anti-mouse IgG (Millipore, Billerica, MA). Membranes were developed using ECL 2 Western Blotting Substrate (Pierce Biotechnology, Rockford, IL) and images were captured using ChemiDoc XRS + system (Bio-Rad Laboratories, Hercules, CA).

\section{T7E1 mismatch-detection assay}

Cleavage activity of AAVS1 and MSTN TALENs, as measured by small deletion/insertion mutations (indels), were quantified by mismatch-recognizing T7E1 as described previously [13]. Briefly, cells transfected with respective TALENs had genomic DNA extracted 48-72 hours post-transfection. Amplification was achieved by using AccuPrime Pfx (Invitrogen, Carlsbad, CA). PCR primers were designed to amplify site surrounding human AAVS1 or human MSTN locus. Specifically, the primer pair for human MSTN binds to Intron 1-2 (hGDF8-F1, 5'TGGAGGGGTTTTGTTAATGG-3') and Intron 2-3 (hGDF8-F2, 5' -TATTGGGTACAGGGCTACCG-3'). The primer pair used for AAVS1 targets the following sites: AS1L-833 F (5' - TTCAGGTTCCGTCTTCCTC CACTC - 3') and AS11-1998R (5' - AACTGACGCA CGGAGGAACAAT-3'). The DNA fragment was purified by using Wizard SV Gel and PCR Clean-Up (Promega, Madison, WI), followed by measurement of DNA concentration by using NanoDrop 2000c spectrophotometer (Thermo Fisher Scientific, Wilmingdon, DE). 600 ng of DNA for MSTN targeting TALEN or 200 ng of DNA for AAVS1 in NEBuffer 2 (New England BioLabs, Ipswich, MA) was then denatured at $95^{\circ} \mathrm{C}$ for 10 minutes, and reannealed slowly using the following temperature program to form DNA heteroduplex if NHEJ occurred: $90 \mathrm{cy}-$ cles of $95-59^{\circ} \mathrm{C}$ with a $0.4^{\circ} \mathrm{C}$ decrease per cycle for 20 seconds, 90 cycles of 59 to $32^{\circ} \mathrm{C}$ with a $0.3^{\circ} \mathrm{C}$ decrease per cycle for 20 seconds, and 20 cycles of 32 to $26^{\circ} \mathrm{C}$ with a $0.3^{\circ} \mathrm{C}$ decrease per cycle for 20 seconds. $0.5 \mathrm{uL}$ T7 Endonuclease I (T7E1; New England BioLabs, Ipswich, MA) was added to the reannealed DNA samples, followed by incubation for 30 minutes. DNA samples were then subjected to electrophoresis on a 2\% TAE agarose gel containing Gel-Red (Biotium, Mayward, CA). The gels were imaged using ChemiDoc XRS + system (Bio-Rad Laboratories, Hercules, CA), and densiometry of DNA bands were quantified using Image software (NIH, Frederick, $\mathrm{MD})$. Mutation frequencies were calculated using the formula: fractional modification $=1-(1-$ (total densiometry of fraction cleaved/total densiometry) $)^{0.5}$ as described [18].

\section{Fluorescence microscopy}

Fluorescence and bright-field images were taken as previously mentioned [13]. In short, images were taken using an inverted Nikon Ti-E microscope equipped with a Xenon lamp (Hamamatsu Photonics Systems, Bridgewater, NJ), a 40 $\times 1.30$ NA objective (Nikon, Tokyo, Japan), and an Evolve 512 EMCCD camera (Photometrics, Pleasanton, CA). The EMCCD camera was cooled to $-80^{\circ} \mathrm{C}$ during imaging. Images were documented and analyzed using NIS-Elements Advanced Research software package (Nikon, Tokyo, Japan).

\section{Statistical analysis}

All data were expressed as mean \pm SEM. Statistical differences were determined by unpaired Student's t-test for two groups using GraphPad Prism 5.0. Statistical significance was defined to be $p<0.05$. 


\section{Additional files}

Additional file 1: The sequence of pTAL10.

Additional file 2: An improved self-cleaving peptide-linked TALEN scaffold for efficient genome editing.

\section{Abbreviations}

PCR: Polymerase chain reaction; TALEN: Transcription activator-like effecto nuclease; GFP: Green fluorescent protein; RVD: Repeat-variable di-residue; MSTN: Myostatin; HA: Hemagglutinin; T7E1: T7 endonuclease I.

\section{Competing interests}

The authors declare that they have no competing interests.

\section{Authors' contributions}

$\mathrm{RH}$ conceived and designed the study. LX and AM carried out the experiments and performed data analysis. AM and $\mathrm{RH}$ wrote the manuscript. All authors read and approved the final manuscript.

\section{Acknowledgments}

Our work is supported by the National Institutes of Health grants R01 AR064241 (R.H.) and R01 HL116546 (R.H.).

\section{Author details}

${ }^{1}$ Department of Cell and Molecular Physiology, Loyola University Chicago Health Sciences Division, Maywood, IL 60153, USA. ²Department of Surgery,

The Ohio State University Wexner Medical Center, Columbus, OH 43210, USA

Received: 20 November 2013 Accepted: 4 September 2014

Published: 10 September 2014

\section{References}

1. Mussolino C, Morbitzer R, Lutge F, Dannemann N, Lahaye T, Cathomen T: A novel TALE nuclease scaffold enables high genome editing activity in combination with low toxicity. Nucleic Acids Res 2011, 39(21):9283-9293.

2. Li T, Huang S, Zhao X, Wright DA, Carpenter S, Spalding MH, Weeks DP, Yang B: Modularly assembled designer TAL effector nucleases for targeted gene knockout and gene replacement in eukaryotes. Nucleic Acids Res 2011, 39(14):6315-6325.

3. Hockemeyer D, Wang H, Kiani S, Lai CS, Gao Q, Cassady JP, Cost GJ, Zhang L, Santiago Y, Miller JC, Zeitler B, Cherone JM, Meng X, Hinkley SJ, Rebar EJ, Gregory PD, Urnov FD, Jaenisch R: Genetic engineering of human pluripotent cells using TALE nucleases. Nat Biotechnol 2011, 29(8):731-734.

4. Cermak T, Doyle EL, Christian M, Wang L, Zhang Y, Schmidt C, Baller JA, Somia NV, Bogdanove AJ, Voytas DF: Efficient design and assembly of custom TALEN and other TAL effector-based constructs for DNA targeting. Nucleic Acids Res 2011, 39(12):e82

5. Moscou MJ, Bogdanove AJ: A simple cipher governs DNA recognition by TAL effectors. Science 2009, 326(5959):1501.

6. Boch J, Scholze H, Schornack S, Landgraf A, Hahn S, Kay S, Lahaye T, Nickstadt A, Bonas U: Breaking the code of DNA binding specificity of TAL-type III effectors. Science 2009, 326(5959):1509-1512.

7. Doyle EL, Booher NJ, Standage DS, Voytas DF, Brendel VP, Vandyk JK Bogdanove AJ: TAL Effector-Nucleotide Targeter (TALE-NT) 2.0: tools for TAL effector design and target prediction. Nucleic Acids Res 2012, 40:W117-W122. Web Server issue.

8. Joung JK, Sander JD: TALENs: a widely applicable technology for targeted genome editing. Nat Rev Mol Cell Biol 2013, 14(1):49-55.

9. Guo J, Gaj T, Barbas CF 3rd: Directed evolution of an enhanced and highly efficient Fokl cleavage domain for zinc finger nucleases. $J \mathrm{Mol}$ Biol 2010, 400(1):96-107

10. Miller JC, Tan S, Qiao G, Barlow KA, Wang J, Xia DF, Meng X, Paschon DE, Leung E, Hinkley SJ, Dulay GP, Hua KL, Ankoudinova I, Cost GJ, Urnov FD Zhang HS, Holmes MC, Zhang L, Gregory PD, Rebar EJ: A TALE nuclease architecture for efficient genome editing. Nat Biotechnol 2011, 29(2):143-148

11. Sun N, Bao Z, Xiong X, Zhao H: SunnyTALEN: a second-generation TALEN system for human genome editing. Biotechnol Bioeng 2014, 111(4):683-691
12. Duda K, Lonowski LA, Kofoed-Nielsen M, Ibarra A, Delay CM, Kang Q, Yang Z, Pruett-Miller SM, Bennett EP, Wandall HH, Davis GD, Hansen SH, Frödin M: High-efficiency genome editing via 2A-coupled co-expression of fluorescent proteins and zinc finger nucleases or CRISPR/Cas9 nickase pairs. Nucleic Acids Res 2014, 42(10):e84.

13. $\mathrm{Xu}$ L, Zhao $\mathrm{P}$, Mariano A, Han R: Targeted myostatin gene editing in multiple mammalian species directed by a single pair of TALE nucleases. Mol Ther Nucleic Acids 2013, 2:e112.

14. Szymczak AL, Workman CJ, Wang Y, Vignali KM, Dilioglou S, Vanin EF, Vignali DA: Correction of multi-gene deficiency in vivo using a single 'self-cleaving' 2A peptide-based retroviral vector. Nat Biotechnol 2004 22(5):589-594.

15. Donnelly ML, Hughes LE, Luke G, Mendoza H, ten Dam E, Gani D, Ryan MD: The 'cleavage' activities of foot-and-mouth disease virus $2 A$ site-directed mutants and naturally occurring '2A-like' sequences. J Gen Virol 2001, 82(Pt 5):1027-1041.

16. Park SH, Chung HK, Kim Do J, Han MR, Park MS, Oh U, Kim HJ, Han BW: Overexpression, crystallization and preliminary X-ray crystallographic analysis of the C-terminal cytosolic domain of mouse anoctamin 1. Acta Crystallogr Sect F Struct Biol Cryst Commun 2011, 67(Pt 10):1250-1252.

17. Miller JC, Holmes MC, Wang J, Guschin DY, Lee YL, Rupniewski I, Beausejour CM, Waite AJ, Wang NS, Kim KA, Gregory PD, Pabo CO, Rebar EJ: An improved zincfinger nuclease architecture for highly specific genome editing. Nat Biotechnol 2007, 25(7):778-785.

18. Bedell VM, Wang Y, Campbell JM, Poshusta TL, Starker CG, Krug RG 2nd, Tan W, Penheiter SG, Ma AC, Leung AY, Fahrenkrug SC, Carlson DF, Voytas DF, Clark KJ, Essner JJ, Ekker SC: In vivo genome editing using a high-efficiency TALEN system. Nature 2012, 491(7422):114-118.

doi:10.1186/1756-0500-7-628

Cite this article as: Mariano et al:: Highly efficient genome editing via 2A-coupled co-expression of two TALEN monomers. BMC Research Notes 2014 7:628.

\section{Submit your next manuscript to BioMed Central and take full advantage of:}

- Convenient online submission

- Thorough peer review

- No space constraints or color figure charges

- Immediate publication on acceptance

- Inclusion in PubMed, CAS, Scopus and Google Scholar

- Research which is freely available for redistribution 